\title{
Téoros
}

Revue de recherche en tourisme

\section{La villégiature forestière en Sagamie}

\section{Jean Désy}

Volume 7, numéro 2, juillet 1988

Villégiature et tourisme

URI : https://id.erudit.org/iderudit/1080410ar

DOI : https://doi.org/10.7202/1080410ar

Aller au sommaire du numéro

\section{Éditeur(s)}

Université du Québec à Montréal

\section{ISSN}

0712-8657 (imprimé)

1923-2705 (numérique)

Découvrir la revue

Citer cet article

Désy, J. (1988). La villégiature forestière en Sagamie. Téoros, 7(2), 17-19.

https://doi.org/10.7202/1080410ar d'utilisation que vous pouvez consulter en ligne.

https://apropos.erudit.org/fr/usagers/politique-dutilisation/ 


\section{Jean Désy*}

\section{La villégiature forestière en Sagamie}

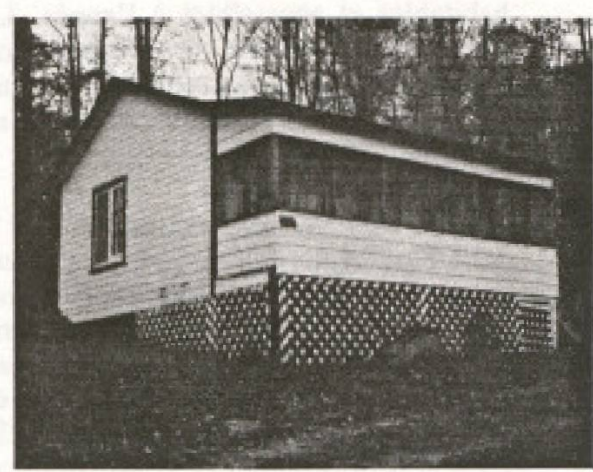

- Jean Désy est géographe et professeur au Departement des sciences humaines a l'Université du Quóbec à Chicoutimi.
Toutes les Municipalités régionales de Comté - ou presque - l'attendent avec impatience. Des milliers de villégiateurs forestiers - réguliers mais surtout illégaux - l'espèrent pour très bientôt, tout comme une multitude de protecteurs de la faune. Et bien sûr, les deux protagonistes majeurs dans ce dossier: les ministères du Loisir, de la Chasse et de la Pêche (MLCP) d'une part, de l'Énergie et des Ressources (MER), d'autre part.

Il s'agit du Plan de développement de la villégiature sur les TNO du Québec (les territoires non organisés), sous la juridiction partagée des MRC, du MER et du MLCP. Un moratoire suspend en effet toute transaction formelle depuis quatre ans entre le MER et tout locataire potentiel d'une parcelle boisée de terre publique pour y bâtir sa résidence secondaire, son chalet ou son camp de chasse et pêche. Ce moratoire, ce fut le compromis accepté par les deux parties en négociation autour d'un enjeu majeur: le mode de répartition des lots de villégiature en forêt publique, qui relèverait avec succès ce périlleux défi de la cohabitation harmonieuse homme-faune.

En Sagamie, plus précisément sur le territoire de la région administrative 02 (Saguenay-Lac-St-Jean), le conflit MLCP $\mathrm{MER}$ revêt une importance singulière, là où se concentrent $21 \%$ des quelque 35000 baux gérés par le MER au Québec en 1988 (environ 7500 dossiers actifs). Mais aussi pour une autre raison: c'est le bureau régional du MLCP qui a en quelque sorte forcé I'ouverture de la ronde nationale de négociations sur ce contentieux, par l'émission d'un "Avis faunique"t contesté par le MER, mais récemment et indirectement endossé par un large consensus d'intervenants au récent sommet québécois sur la Faune (thème 2, sujet 4: "L'accessibilité à l'utilisation de la faune"). Enfin, pour trois des quatre MRC de la région anxieuses de voir leur schéma d'aménagement endossé par le gouvernement, ce plan de développement de la villégiature représente le plus gros obstacle qui bloque tout le processus, cas sans doute similaire à celui de dizaines de MRC au Québec.

Je vous propose donc une démarche d'observateur toute simple: d'abord présenter les intervenants majeurs du dossier et leurs positions respectives: MER, MLCP, MRC et $\mathrm{ZEC}$; circonscrire en second lieu les grandes caractéristiques de l'offre et de la demande régionales en matière de villégiature forestière; esquisser enfin la démarche- type de l'aspirant-locataire, histoire de démonter les pièces d'une mécanique administrative assez complexe, mais fort instructive.

\section{Le "socio-système" de la villégiature forestière}

\section{Le MER}

Énergie et Ressources, c'est le ministère-clé de tout le territoire forestier québécois, celui qui doit concilier l'appétit féroce de papetières, la grogne des villégiateurs-sportifs en quête de terres giboyeuses, et enfin la survie de la flore et de la faune... pendant et après satisfaction de ces deux blocs sociaux de prédateurs!

C'est lui qui a absorbé l'impact des politiques d'ouverture et d'accessibilité de la forèt vers la fin des années "70, après le long règne des clubs privés de chasse et pêche, jumelée à la chasse-gardée des papetières sur leurs concessions forestières. Quelques dates:

1977: Politique d'accessibilité des terres publiques par le MTF aux citoyens et décrets afférents $(27-40,27-41,27-42)$.

1978: "Déclubage"" (opération gestion faune) et création des ZEC par le MLCP; dix seront décrétées au Saguenay-Lac-St-Jean.

1978-82: Opération de régularisation des occupations illégales ("squatters") par le MER. En Sagamie, on traite plus de 1000 dossiers de ce type qui s'ajoutent aux 2150 réguliers de 1978 (17,7\% du Québec) et aux demandes courantes.

1982-84: La demande croît toujours sans fléchir et atteint 5566 dossiers actifs en Sagamie sur plus de 26000 pour le Québec, totalisant $21 \%$ du parc de villégiature forestière national.

1984: Moratoire imposé sur les baux, à la suite des divergences de vue entre le MLCP et le MER sur le mode de fixation de la capacité de charge du territoire.

1984-88: En Sagamie toujours, mise en filière de 2300 demandes fermes et réapparition significative des occupants illégaux.

\section{Le MLCP}

Gestionnaire des ZEC et de la faune sur les territoires forestiers, le MLCP est souvent confronté au MER sur qui s'exercent les puissants "lobbies" papetiers et sportifs pour obtenir faveurs et largesses de l'État. Le MLCP privilégie une approche à petite 
Echelle du territoire faunique, c'est-à-dire embrassant de larges écosystemes forestiers dont le potentiel biologique doit pouvoir soutenir la demande de chasse et pêche. Le MER, de son côté, dit vouloir rendre accessible aux citoyens un maximum de lacs et de rivières, sur la base d'une analyse à la pièce de chaque plan d'eau.

En bref, voici l'essentiel de l'avis faunique du bureau sagamien du MLCP, tel que résumé et adopté majoritairement par les intervenants du Sommet sur la faune:

1) Maintenir un minimum de territoire public libre d'affectation.

2) Établir une stratégie de gestion de la faune en territoire libre.

3) Conserver les secteurs les plus accessibles pour les activités quotidiennes.

4) Orienter les camps de chasse et pêche vers les secteurs plus éloignés et la villégiature commerciale (permissionnaires) vers les secteurs plus rapprochés.

5) Favoriser le développement de villégiature par póles.

6) Limiter le nombre d'occupations en villégiature en fonction du potentiel faunique là où la péche et la chasse sont des activités dominantes.

7)

Orienter la villégiature de loisir vers les plans d'eau à potentiel de péche faible.

8) Maintenir temporairement le moratoire sur le développement de la villégiature.

\section{Les MRC}

Grande déception du côté des MRC: le droit de gestion du MER l'emporte, et de loin, sur le droit d'aménagement que leur confere la loi 125 ! C'est ainsi que l'approche plutôt conservationniste de plusieurs d'entre elles. assez proche de celle du MLCP, $s^{4}$ est butée aux résistances soutenues du MER, qui bloque l'adoption globale de nombreux schémas d'amenagement. La MRC Domaine-du-Roy (secteur Roberval) profite toutefois d'une experience pilote régionale, c'est-à-dire d'un plan de développement MER de la villégia- ture sur TNO. Ce plan encore confidentiel propose trois types d'espace: a) de villégiature; b) d'accès public et c) de conservation, ce qui augure déjă d'une ouverture intéressante de ce ministère a de nouvelles propositions. Une démarche similaire est en cours a la MRC Lac-St-Jean-Est.

Dès l'adoption des schếmas par le gouvernement, les MRC auront à légiférer sur les règlements de zonage, lotissement et construction, propres à ce secteur, ce que prépare d'ailleurs la MRC de Lac-St-Jean-Est, dont le schéma est en vigueur.

\section{Les ZEC}

Détentrices depuis 1978 de droits de gestion de la faune sur des territoires forestiers délimités, et responsables de ce mandat devant le MLCP, les ZEC n'ont guère encouragé jusqu'à maintenant l'installation de chalets de villégiature sur leurs territoires. Pour une raison bien simple: de $75 \%$ à $80 \%$ des administrateurs de ZEC sont d'ex-membres de clubs privés d'avant 1978 et $50 \%$ d'entre eux y siègent depuis ce temps...

Les quelque 550 à 600 membres des 62 conseils de ZEC à travers le Québec tentent donc de maintenir, pour quelques années encore, le privilège soi-disant acquis de père en fils, de jouissance exclusive de tel lac, de tel segment de rivière, de telle aire de chasse. Néanmoins, pour toutes les ZEC du Québec, en 1985 , on dénombrait 7200 chalets privés qui auraient accueilli au moins 81000 personnes ${ }^{(1)}$ et jusqu'au moratoire de 1984 , le MER répondait régulièrement à la demande sur ces territoires publics, au grand dam des gestionnaires. Les dix ZEC de la région 02 abritaient 2757 chalets en 1985 , y accueillant 10500 personnes. Depuis, les squatters y ont sans doute moins proliféré que sur les TNO sans surveillance, mais leurs constructions y demeureront pour longtemps, rarement condamnées à disparaître par quelqu'autorité que ce soit; on les légalise plutớt, question de coûts. .

\section{L'offre et la demande globale de villégiature forestière}

En date du 11 mai 1988, le bureau régional du MER dénombrait 7898 occupations de "villégiature" au sens large, ainsi réparties par MRC (voir tableau ci-dessous).

\section{TABLEAU}

L'offre et la demande globales de villégiature forestitre

\begin{tabular}{llcc}
\hline MRC & Le Fjord du Saguenay & 3.230 & $40,9 \%$ \\
MRC & Maria-Chapdelaine & 2097 & $26,5 \%$ \\
MRC & Le Domaine-du-Roy & 1342 & $17,0 \%$ \\
Hors MRC & (MBJ + Nouveau-Québec) & 671 & $8,5 \%$ \\
MRC & Lac-St-Jean-Est & 558 & $7,1 \%$ \\
TOTAL: & & 7898 & $100,0 \%$ \\
\hline
\end{tabular}

On peut aussi répartir l'offre en trois zones concentriques:

1- La zone de villégiature résidentielle, en territoire municipal sur terres publiques. Le MER régional y gère près de 1500 dossiers de location (baux de 30 ans), notamment dans certains secteurs du lac Kénogami et du lac St-Jean (Baie Cascouia et Pointe Racine). La valeur moyenne de ces résidences secondaires se situe autour de $20000 \$$, pour la plupart habitables et accessibles à l'année. Un pourcentage minime des terrains est revendu aux locataires moyennant certaines conditions d'aménagement et de gestion.

2- La zone de villegiature rustique. Pour le MLCP, c'est la couronne à potentiel faunique exceptionnel, accessible sur une base quotidienne en voiture, pour un trajet variant de 1 heure à 2 heures 30 à partir des agglomérations (50 à $100 \mathrm{~km}$ de limites de l'écoumène). Pour le MER, c'est la zone des baux de courte durée ( 8 ans), renouvelables et transférables, totalisant plus de 5000 dossiers, les deux-tiers des logements forestiers. Leur évaluation moyenne est de $12000 \$$. Le rachat y est impossible.

3- La zone éloignée, celle des camps de chasse et pêche temporaires et rudimentaires ( $5000 \$$ en moyenne, mais dont le transport fait vite grimper les coûts globaux de construction). Les baux de quatre ans sont également renouvelables et transférables, tous appliqués à des camps hors pourvoiries. Le séjour s'impose habituellement en raison du fort eloignement ou du temps d'accès supérieur à $2 \mathrm{~h} 30$ heures. Le MER gère près de 700 de ces dossiers, y compris ceux du secteur au sud de la région 10 (Ungava) compris entre le 70 et 76 eme méridien.

L'accessibilité croissante de l'espace forestier sillonné depuis vingt ans par un réseau de "'maîtres chemins" bien entretenus, souvent l'hiver, figure comme premier facteur d'explosion "résidentielle" en forêt. Dans les $\mathrm{ZEC}$, on relève en 1985 que " $73 \%$ des lacs et $91 \%$ de leur superficie en eau sont considérés accessibles" (...) et que le territoire proprement dit "iserait accessible à plus de $86 \%(.$.$) , et davantage les ZEC et lacs$ des régions $02,01,03$ et $04^{(2)}$.

A défaut de statistiques mensuelles de fréquentation de ces milliers de chalets, les témoignages nous fournissent le biorythme annuel probable à ce chapitre:

- fin mai, réfection des chemins défoncés, dégel du mouvement centrifuge urbain vers la forêt;

- St-Jean - Fête du travail, occupation probablement moyenne, réduité par l'invasion des moustiques et les activites touristiques régionales et extra-régionales; 
- septembre, période creuse de transition été-automne;

- octobre, reprise pour la saison de la chasse;

- nov. - déc, période charnière automnehiver. Baisse maximale;

- janv. - avril, reprise modérée pour sports d'hiver motorisés et séjours de week-end à partir du réseau de routes majeures entretenues:

- avril - mai, période creuse de soudure printemps-été, en raison surtout des routes peu pratiquables.

Au chapitre de la demande, le clair-obscur règne souverainement dans les officines ministérielles et MRCéennes: les programmes informatiques ne seraient pas conçus pour pouvoir produire l'information sur l'origine géographique, sociale ou professionnelle des villégiateurs. On émet de vagues intuitions sur deux sources émettrices majeures: les centres urbains et les agglomérations agro-forestières périphériques à l'écoumène. On estime à $85 \%-90 \%$ la proportion de régionaux détenteurs de baux dans les zones 1 et 2, Québec et Montréal comblant la différence. Ce serait l'inverse pour la zone 3 , où $90 \%$ des "villégiateurs" sont des extrarégionaux, pour des flux globalement minimes toutefois.

L'enquête panquébécoise réalisée par Gilles Laterrière en 1983 pour le MLCP, ne fournit aucune ventilation régionale sous ce rapport. Le portrait national coïncide-t-il avec le portrait régional, c'est-à-dire que "toutes les catégories sociales ont un accès relativement important à la villégiature privée, bien qu'on observe certaines inégalités'? (surreprésentation des 12-20 ans et sous-représentation des 20-34, participation plus forte des hommes par rapport aux femmes, notamment veuves, séparées, divorcées, surreprésentation des travailleurs syndiqués,...). Réponse d'autant plus malaisée que les villégiateurs forestiers de l'étude réalisée en 1981 , ne semblent constituer que $5,7 \%$ de l'ensemble des 302300 menages propriétaires d'une résidence secondaire ${ }^{(3)}$.

On y découvre cependant que la Sagamie se retrouve au deuxième rang des régions administratives en ce qui concerne la proportion des ménages-propriétaires d'une résidence secondaire $(17533$, soit $24,1 \%)$, très près de l'Abitibi et de la Côte Nord.

Notre propre inventaire des résidences secondaires, déjà trop âgé $(1981)^{(4)}$, exclut les territoires non organisés (TNO) où se localise l'essentiel de la villégiature rustique. On signalait par contre que les salaires relativement élevés que gagnent les travailleurs de la grande entreprise permettent à ceux-ci de s'imposer dans la répartition de ces biens fonciers (intra-municipaux). Rien ne permet de croire, au contraire, à un renversement du tableau pour l'occupation du territoire forestier hors municipalité.
Mais avouons-le, des analyses fines et récentes font cruellement défaut, malgré la disponibilité relative des statistiques compilées par le MER et les MRC.

\section{La démarche d'un aspirant-locataire}

St-Léon-de-Chicoutimi: à vol d'oiseau, 15 $\mathrm{km}$ au NNE d'Alma, $43 \mathrm{~km}$ au NO de Chicoutimi; petit village " agro-récréatif' de 800 habitants, installé sur les rives du lac Léon, un lac de villégiature assez actif. Claude B. enseignant au secondaire à Alma, aspire à la tranquilité d'un espace vierge, avec sa famille. Des excursions précédentes chez un ami construit sur les rives de la rivière Péribonka, lui donnent la piqure d'un camp rustique en forêt. Leur choix se porte sur un emplacement situé à $15 \mathrm{~km}$ au nord de StLéon, en bordure de la Péribonka, dont $\mathrm{L}^{\dagger}$ accessibilité est variable selon les saisons:

- en été, on rejoint le camp en une heure, par terre et par eau à partir du lac Tchitagama;

- en hiver, une randonnée de 30 minutes en motoneige sur neige et sur glace permet un accès facile et rapide; sinon, c'est un grand détour de deux heures trente de routes malaisées par Falardeau et la ZEC Boîteuse.

Première démarche administrative: le bureau régional du MER d"Alma. Au bout de deux à trois semaines, réponse favorable pour un bail court terme ( 8 ans), sur un terrain de $3792 \mathrm{~m}^{2}$. Deuxième démarche administrative: le bureau de la MRC du Fjord-duSaguenay à Chicoutimi pour le permis de construction de 30\$. Problème: la MRC exige une superficie minimale de $4000 \mathrm{~m}^{2}$ ! Retour au MER: " "que la MRC se plie à nos règlements".... Finalement, on consentira à l'agrandissement.

Claude B. érige donc son camp sur un carré de $20^{\prime} \times 20^{\circ}$, en contreplaqué pré-peint à l'extérieur et pré-fini à l'intérieur. Il bùche lui-même la charpente sur un boisé environnant, fait scier son bois par un voisin équipé d'un moulin à scie artisanal, récupère à peu de frais portes et fenetres, n'installe ni plomberie sophistiquée ni électricité, mais un bon poêle à bois et une toilette chimique. Total de l'investissement: de $3500 \$$ à $4000 \$$, excluant bien sûr les coûts d'une main-d'oeuvre amicale gratuite. Coût annuel de location au MER: 131\$. Coût annuel de la taxe MRC: $4,10 \$$..

Claude B. rentabilise bien son investissement: il se rend environ deux fois par semaine à son chalet au cours des quatre mois d'hiver pratiquables, de la mi-décembre à la mi-avril, en plus de la semaine de relâche. Après la fonte des glaces (mai-juin), le rythme se limite aux fins de semaine et aux congés. Mais l'été, c'est le calme plat de ce côté, abandonnant le territoire aux mouches noires et aux "frappe-à-bord", leur preférant les voyages... jusqu'au temps des bleuets (fin aoult). Puis c'est la reprise en octobre jusqu'aux glaces, pour la chasse et la détente...

... La détente que viendront peut-être sérieusement perturber par périodes, les vols en rase motte des F-18, en quête d'un champ de tir académique, projeté non loin de son chalet par la Défense nationale... D'ailleurs, depuis deux ans, tout permis est suspendu dans ce secteur pour des imperratifs hautement stratégiques.

\section{Une face cachée de la forêt}

Une puissante force centrifuge amène donc les régions du Québec depuis dix ans, de l'écoumène vers la forèt. Les papetières et les scieries ne sont plus reines et maîtresses de ce royaume, pas plus que chasseurs et pêcheurs ne détiennent le monopole de cet espace et de ses ressources. Les villégiateurs et plein-airistes l'occupent maintenant avec énergie, multiplient la pression sur l'occupation et l'affectation de terres publiques, aux juridictions si nombreuses et aux convoitises si farouches.

Nous devons malheureusement regretter l'indigence des recherches approfondies sur ce phénomène aux allures explosives qui doit depasser le stade des statistiques sommaires et des descriptions qualitatives et générales. Des enjeux majeurs se dessinent pour les prochaines années sur ce territoire: utilisation polyvalente des ressources, "syndrôme urbano-forestier" , gestion sociale élargie de ce patrimoine collectif qu'est la forêtt québécoise. Aux chercheur-e-s des Sciences humaines de s'y attaquer! $f$
Notes explicatives

(1) Oúbec-MLCP, Les zones d"explohtation contro láe. Bilan 1985.... Quóbec, mai 1987, 23 me édition. p. 12 .

(2) Op, cit., p. 16.

(3) Québec, MLCP, par Gilles Laterrière, La place de la villégiature privée dans les loisirs des Quobbécois et dans leur vie sociale et économique. Quebec, déc. 1983, p. 11 at 15.

(4) Les laboratoires de góographie de I'UOAC. Atlas rogional du Saguenay-Lac-St-Jean, Chicoutimi, Gadtan Morin éditeur, planche C-9, Les rásidences secondaires, 1981. 